\title{
Proton Magnetic Resonance Spectroscopy Study of Brain Metabolism in Obstruc- tive Sleep Apnoea Syndrome before and after Continuous Positive Airway Pressure Treatment.
}

Caterina Tonon, MD¹; Roberto Vetrugno, MD, PhD²; Raffaele Lodi, MD; Roberto Gallassi, MD²; Federica Provini, MD²; Stefano lotti, PhD'; Giuseppe Plazzi, MD²; Pasquale Montagna, MD²; Elio Lugaresi, MD²; Bruno Barbiroli, MD

${ }^{I}$ Dipartimento di Medicina Clinica e Biotecnologia Applicata \& Dipartimento dell'Area Radiologica, Policlinico S. Orsola-Malpighi, University of Bologna; ${ }^{2}$ Dipartimento di Scienze Neurologiche, University of Bologna, Bologna, Italy

Study Objectives: Obstructive sleep apnoea syndrome (OSAS) causes sleep related oxygen desaturation, excessive daytime sleepiness (EDS), and cognitive impairment. The role of hypoxic brain damage, sleep fragmentation, and the associated comorbidities (hypertension, vascular disorders) in the pathogenesis of cognitive deficits remains controversial. The aim of this study was to evaluate the cerebral metabolism of OSAS patients in vivo before and after CPAP treatment.

Design and Patients: Fourteen OSAS patients without cardiovascular or cerebrovascular impairment underwent the same protocol before and after 6 months of CPAP including: overnight videopolysomnography (VPSG), Multiple Sleep Latency Test (MSLT), and within the next 2 days neuropsychological and ${ }^{1} \mathrm{H}-\mathrm{MRS}$ evaluations. Single voxel ${ }^{1} \mathrm{H}-\mathrm{MRS}$ was performed in the parietal-occipital cortex, and absolute concentrations of $\mathrm{N}$-acetyl-aspartate (NAA), creatine, and choline were measured, acquiring spectra at multiple echo-times and using water as internal standard. Ten matched controls were also studied.

Results: OSAS patients had a mean RDI of $58 / \mathrm{hr}$, a mean arousal index of $57 / \mathrm{hr}$, and a mean nadir SpO2 of $71 \%$. Before CPAP, all patients showed a normal global cognitive functioning, with only a small number of pathological tasks in working memory and attention tests in a minority of patients.
CPAP therapy was effective in resolving sleep apnoea and normalizing sleep structure, and improving EDS and neuropsychological alterations. Before CPAP treatment cortical [NAA] in OSAS (11.86 $\mathrm{mM} \pm 0.80$, mean $\pm S D)$ was significantly lower than in controls $(12.85 \pm 0.93 ; P=0.01)$ and positively correlated with minimum SpO2 during sleep $(r=0.69 ; P$ $=0.006)$ and MSLT scores $(r=0.62 ; P=0.01)$. Cortical $[N A A]$ reduction persisted after therapy $(11.94 \pm 1.33 ; P=0.87$ versus pre-CPAP).

Conclusions: OSAS patients have cortical metabolic changes consistent with neuronal loss even in the absence of vascular comorbidities. Metabolic changes persisted after CPAP in the absence of EDS, nocturnal arousals, and major cognitive deficits, likely related to hypoxic damage prior to CPAP treatment.

Keywords: Obstructive sleep apnoea syndrome, proton magnetic resonance spectroscopy, $\mathrm{N}$-acetyl-aspartate, nasal continuous positive airway pressure, polysomnography, chronic hypoxia, follow-up.

Citation: Tonon C; Vetrugno R; Lodi R et al. Proton magnetic resonance spectroscopy study of brain metabolism in obstructive sleep apnoea syndrome before and after continuous positive airway pressure treatment. SLEEP 2007;30(3):305-311

memory, executive and motor functions have been documented in OSAS. ${ }^{5,6}$ However, conflicting findings emerge from a metaanalysis on the neuropsychological effects of obstructive sleep apnea in untreated patients. General intelligence, including basic verbal and visual-perceptual abilities were unaffected, whereas vigilance, executive functions, and motor coordination were moderately to markedly impaired. The effect of OSA on visual skills, motor skills, and memory functioning was inconsistent. ${ }^{6}$

Continuous positive airway pressure (CPAP) applied through a nasal mask is the first line of treatment in OSAS. By preventing upper airway collapse, CPAP normalizes sleep patterns and EDS and improves quality of life. ${ }^{7,8}$ The positive impact of CPAP has mainly been detected on vigilance, attention, and memory deficits that are likely related to sleep fragmentation. ${ }^{8,9}$ Other neuropsychological changes, especially in the executive, motor, and visuoconstructive domains, tend to persist despite CPAP treatment, probably due to irreversible hypoxemic damage. ${ }^{8,9}$

Neuropsychological and functional imaging studies addressing the problem of cognitive deterioration in OSAS have rarely considered the impact of comorbidities such as hypertension, vascular disease, and diabetes mellitus in determining neurological dysfunction. This has often led to inconsistent and controversial results.

Proton magnetic resonance spectroscopy $\left({ }^{1} \mathrm{H}-\mathrm{MRS}\right)$ is a sensitive, non-invasive technique that assesses and monitors biochemical changes in brain areas of interest. This technique allows direct quantification of brain metabolites, including N-acetylaspartate (NAA). Most studies have indicated that NAA is a putative neu- 
ronal marker ${ }^{10}$ located in neurons and neuronal processes. Reduced NAA concentration is typically seen in neurodegenerative, inflammatory, or vascular disorders. ${ }^{10}$ The partial reversibility of NAA deficit after therapy ${ }^{11}$ or during recovery from acute brain pathology ${ }^{12}$ indicates that reduced brain NAA may be not only related to neuronal loss, but also to neuronal dysfunction.

${ }^{1} \mathrm{H}-\mathrm{MRS}$ studies on OSAS patients have assessed cerebral metabolism in different brain regions, yielding inconsistent results. A significant decrease in the NAA/Cho ratio associated with slight decrease in NAA/Cr, and increase in $\mathrm{Cho} / \mathrm{Cr}$ was found in the posterior periventricular white matter. ${ }^{13}$ However, a later study detected a significant reduction in both NAA/Cr and $\mathrm{Cho} / \mathrm{Cr}$ in the frontal white matter. ${ }^{14}$ Investigation of the left hippocampus revealed increased $\mathrm{NAA} / \mathrm{Cr}$, suggesting reduced creatine in OSAS patients. ${ }^{15}$ The inconsistent results of these studies may be related to both MRS methodological differences and the clinical heterogeneity of the patient population recruited.

We used ${ }^{1} \mathrm{H}-\mathrm{MRS}$ to evaluate brain metabolism in OSAS patients strictly selected for the absence of cardiovascular and cerebrovascular comorbidities. Absence of comorbidities was required to avoid potential confounding factors and to evaluate only brain metabolic features directly resulting from recurring apnoea/hypopnea during sleep.

The MRS acquisition protocol was designed to assess brain concentrations of NAA, choline, and creatine ${ }^{16}$ to avoid uncertainties in interpreting metabolite ratios, namely possible changes in creatine concentration. ${ }^{15}$ The same study protocol applied before and after 6 months of CPAP included videopolysomnography (VPSG) with Multiple Sleep Latency Test (MSLT), neuropsychological testing, and ${ }^{1} \mathrm{H}-\mathrm{MRS}$ assessment.

\section{METHODS}

\section{Subjects}

Fourteen OSAS patients with mean body mass index (BMI) of $32.3 \pm 4.6$ and EDS, as assessed by means of non-structured clinical interview, were recruited from the Sleep Disorders Centre of the Dipartimento di Scienze Neurologiche of the University of Bologna, Italy. To qualify for the study, subjects had to have an EDS (Epworth Sleepiness Scale, ESS) score $\geq 10$; normal wake and sleep systolic and diastolic blood pressure; no diabetes mellitus or hyperlipidaemia; no cardiovascular disorders; and no history or current evidence of drug abuse or neurological disease. Patients with abnormalities on neurological examination or structural brain lesions on brain CT and conventional MRI were excluded. Patients were asked to refrain from drugs throughout the study and were drug free at the time of examination. Ten age- and sex-matched healthy controls were recruited on the basis of the same exclusion criteria used for the patients. Healthy subjects had mean BMI 25 \pm 2 , range 22-28; mean diastolic (DBP) $72 \pm 4$, range 60-82; systolic blood pressure (SBP) 120 4 , range 105-133; normal ESS scores; and normal brain MRI.

The approval from the Policlinico S.Orsola Hospital Ethics Committee and written informed consent from each participant were obtained.

\section{Study Design}

All OSAS patients completed a standardized study protocol including: a diagnostic overnight VPSG with MSLT the follow- ing day; neuropsychological tests (performed between 15:00 to 18:00) and ${ }^{1} \mathrm{H}-\mathrm{MRS}$ (performed between 14:00 to 16:00) evaluations within the following 2 days; VPSG with CPAP titration; and subsequent MSLT within one week. The same protocol study was performed again after 6 months of CPAP treatment.

\section{Sleep Study}

\section{Nocturnal VPSG Procedures}

A diagnostic overnight VPSG was carried out in all OSAS patients, after an adaptation night in the sleep laboratory. VPSG with CPAP titration was then performed, and patients were prescribed CPAP. Patients were reevaluated with VPSG 6 months later, and compliance with prescribed CPAP treatment was evaluated by means of self-reported use, expressed as the average time with CPAP in relation to total night time spent in bed, assessed with a patient diary.

VPSG data were analysed in 30-s epochs according to Rechtschaffen and Kales, ${ }^{17}$ and sleep structure and sleep efficiency (percentage of total sleep period spent asleep) were calculated. Snoring was evaluated by means of tracheal microphone. An abnormal breathing event during sleep was defined as apnoea (a complete cessation of airflow lasting $\geq 10$ seconds) or hypopnoea (a discernible reduction of airflow accompanied by a decrease of at least $4 \%$ in oxyhaemoglobin saturation $\left(\mathrm{SpO}_{2}\right) \cdot{ }^{18-20}$ Obstruction was confirmed by respiratory effort recorded by thoracic-abdominal strain gauge, increased negative endoesophageal pressure by intraesophageal balloon, and intercostalis electromyogram. The average number of apnoea-hypopnoea episodes per hour of sleep was calculated as the respiratory disturbance index (RDI). Transient arousals were scored as visible EEG arousals lasting $\geq 2$ seconds and not associated with any stage/state change in the 30-s epoch scoring. ${ }^{21}$ The arousal index (AI) (number of arousals per hour of sleep) was calculated. Mean $\mathrm{SpO}_{2}$ value, mean diastolic (DBP) and systolic blood pressure (SBP), and heart rate (HR), and their minimum and maximum values were calculated in relation to total sleep period (TS) for each patient.

\section{Objective and Subjective Sleepiness Assessment}

Diagnostic overnight VPSG, CPAP titration, VPSG, and CPAP retitration VPSG after 6 months of CPAP treatment were all followed by an MSLT to evaluate objective sleepiness. ${ }^{22}$ Each MSLT (5 sessions) was performed beginning not earlier than 2 hours after morning awakening.

Before diagnostic VPSG and after 6 months of CPAP treatment the Epworth Sleepiness Scale (ESS) ${ }^{23}$ for measuring subjective daytime sleepiness was administered to each patient.

\section{Neuropsychological Evaluation}

At baseline before and 6 months after CPAP all patients were administered a battery of neuropsychological tests assessing: 1) global cognition by Mini Mental Status Examination (MMSE), ${ }^{24}$ corrected for age and education according to Italian standardization (cut-off: 23.8) 25 $^{25}$ and the Brief Mental Deterioration Battery (BMDB) with its Final Result (FR) ${ }^{26,27}$ (normal values above 0); 2) memory by 15 Rey's words with immediate and delayed recall (verbal learning of word lists and delayed free recall), immediate visual memory (visual recognition), digit span forward (immediate verbal memory) and backwards (working memory 

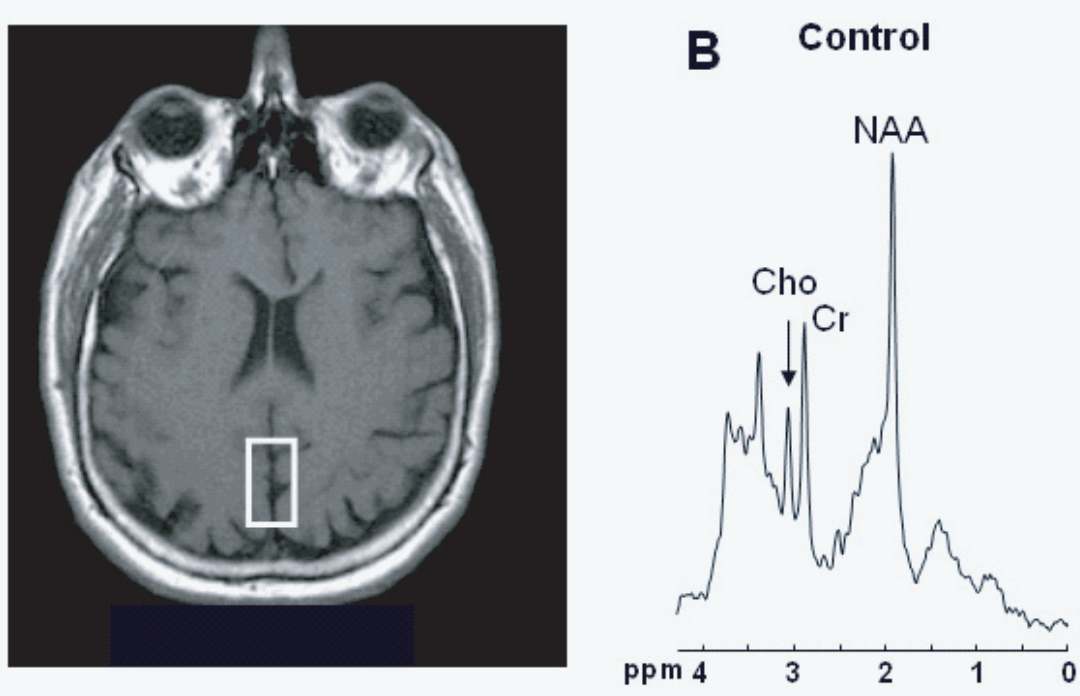

OSAS patient

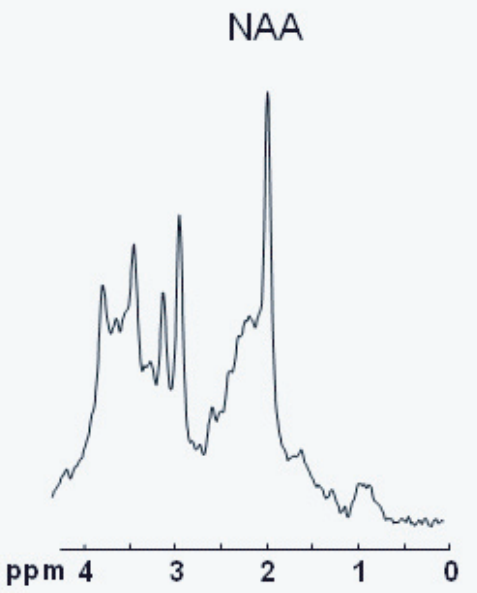

Figure 1-A) Localization of medial brain parietal-occipital grey matter volume of interest (VOI). B) Proton MR spectra from an OSAS patient (case no. 4) compared to a sex- and age-matched control ( $\mathrm{TE}=35 \mathrm{~ms}$; $\mathrm{TR}=4000 \mathrm{~ms})$. The NAA (N-acetyl-aspartate) peak is lower in the patient.

task), Corsi's cube span (immediate visuo-spatial memory); 3) executive functioning by phonemic fluency (verbal production ability), analogies (logical-deductive ability), Weigl's sorting test (categorical thinking); 4) constructional praxis by copy design (visuo-construction ability); 5) attention via attention barrage by visual selective attention task and 6) psychomotor performance by computerized auditory and visual simple and complex reaction times (vigilance and selective attention tasks), finger tapping test (manual dexterity), "watch test" (sustained attention). These tests were chosen because they explore the cognitive domains previously assessed in the literature in OSAS patients. ${ }^{6}$ The same tests were standardized in the Italian population and in our own normal control samples ${ }^{26,27}$ and have been adopted in other sleep dysfunction studies. ${ }^{28}$

\section{${ }^{1} \mathrm{H}$-MRS Protocol}

Brain MR imaging and spectroscopy studies were carried out on a 1.5 Tesla clinical whole body magnet (General Electric Medical Systems, Milwaukee, WI, USA) with a quadrature birdcage headcoil (25 cm of diameter), at S. Orsola-Malpighi Hospital, Bologna. ${ }^{1} \mathrm{H}-\mathrm{MRS}$ was conducted from 14:00 to 16:00 in all patients. The MRI protocol consisted of axial T1-weighted Spin-Echo (SE) images (Echo Time, TE=14 ms; Repetition Time, TR=500 ms; $\mathrm{FOV}=24 \mathrm{~cm}$; 20 slices of $4 \mathrm{~mm}$ of thickness; gap=1mm) and axial fluid-attenuated inversion recovery (FLAIR) ( $\mathrm{TE}=97 \mathrm{~ms}$, $\mathrm{TR}=8000 \mathrm{~ms}, \mathrm{FOV}=24 \mathrm{~cm}, 20$ slices of $5 \mathrm{~mm}$ of thickness, gap $=1$ $\mathrm{mm}$ ). Single voxel spectroscopy was performed with the point resolved spectroscopy sequence (PRESS). A volume of interest (VOI) of $18 \mathrm{~cm}^{3}$ was placed in the brain medial parietal-occipital grey matter (Figure 1A). The VOI included both parietal and occipital cortex. The occipital cortex was included for 2 reasons: a) there is some evidence that there is a reduction in thickness in the occipital cortex of OSAS patients, ${ }^{29}$ b) to obtain a good quality MRS data set with a scan protocol duration below 1 hour (i.e. high signal to noise ratio), we selected a relatively large VOI, and part of the occipital cortex had to be included in the VOI.

The water signal was suppressed by the CHESS (Chemical Shift Selective) sequence. Absolute quantification was made by applying the method developed by the "EEC Concerted Action
BIOMED I programme". ${ }^{16}$ This method uses water as internal standard and involves the acquisition of spectra at long TRs for both metabolites and water (to separate brain from cerebrospinal water). ${ }^{16,30}$ Absolute concentrations of NAA, creatine-phosphocreatine $(\mathrm{Cr})$, and choline (Cho) were measured by acquiring spectra at 5 echo times $(\mathrm{TE}=35,70,100,144,288 \mathrm{~ms} ; \mathrm{TR}=4$ $\mathrm{s}$; number of acquisitions $=32$ ). Brain water content from the selected VOI was obtained by acquiring single spectra, without water suppression, at $\mathrm{TE}=20,30,40,50,60,80,100,300,600$, $900,1000 \mathrm{~ms}(\mathrm{TR}=15 \mathrm{~s})$. The water peak area was calculated for each spectrum with Fourier transform and the Lorentzian fit (Levemberg-Marquardt) method. Data were processed with a bi-exponential fit to separate brain from cerebrospinal water to extrapolate the value of cerebral water signal intensity at $\mathrm{TE}=$ 0 . Peak areas for NAA at $2.02 \mathrm{ppm}$, for $\mathrm{Cr}$ at $3.03 \mathrm{ppm}$, and for Cho at $3.22 \mathrm{ppm}$ were calculated using the time domain fitting program AMARES/MRUI (htpp://carbon.uab.es/mrui), as previously described. ${ }^{31}$ Spectroscopic analyses were performed by one spectroscopist (R. L., 15 years' experience) who was blinded to the subject's condition.

\section{Statistical Analysis}

For the ${ }^{1} \mathrm{H}-\mathrm{MRS}$ data, Student's unpaired t-test was used to compare OSAS patients at baseline and healthy controls. The OSAS patients before and after CPAP were compared using Student's paired t-test. Linear regression analysis was used to calculate correlation coefficients. Statistical significance was taken as $\mathrm{P}<0.05$. For the neuropsychological data the nonparametric test for 2 dependent samples of Wilcoxon with 0.01 level of significance was used.

\section{RESULTS}

\section{Sleep Studies}

Demographic and clinical/PSG characteristics of OSAS patients are shown in tables 1-2. At baseline all OSAS patients had an ESS score $\geq 10$. Mean wake SBP/DBP and HR (average of 3 consecutive determinations at 10-minute intervals) were $130.7 / 84.4$ 
Table 1-Main demographic and clinical data from OSAS patients.

\begin{tabular}{lccccccc}
$\begin{array}{l}\text { Case } \\
\text { n\%/Sex }\end{array}$ & Age & BMI & $\begin{array}{c}\text { Snoring } \\
\text { duration } \\
\text { (yrs) }\end{array}$ & $\begin{array}{c}\text { Sleep } \\
\text { apnoea } \\
\text { duration } \\
\text { (yrs) }\end{array}$ & $\begin{array}{c}\text { EDS } \\
\text { (yrs) }\end{array}$ & MMSE & BMDB \\
& & \multicolumn{7}{c}{$\begin{array}{c} \\
\text { 1/M }\end{array}$} & 54 & 33.5 & 34 & 10 & 2 & 27.97 & 2.35 \\
$2 / \mathrm{M}$ & 51 & 32.5 & 35 & 3 & 3 & 29.97 & 3.01 \\
$3 / \mathrm{M}$ & 54 & 25.8 & 15 & 9 & 2 & 29.97 & 1.54 \\
$4 / \mathrm{M}$ & 57 & 31.8 & 10 & 4 & 2 & 28.97 & 2.52 \\
$5 / \mathrm{M}$ & 44 & 32.7 & 30 & 10 & 1 & 28.31 & 2.92 \\
$6 / \mathrm{M}$ & 46 & 33.4 & 30 & 12 & 2 & 29.62 & 2.58 \\
$7 / \mathrm{M}$ & 50 & 37.3 & 32 & 1 & 1 & 28.99 & 2.37 \\
$8 / \mathrm{M}$ & 45 & 29.6 & 15 & 4 & 4 & 28.21 & 2.16 \\
$9 / \mathrm{M}$ & 57 & 27.8 & 40 & 2 & 2 & 28.74 & 1.86 \\
$10 / \mathrm{M}$ & 54 & 27.1 & 34 & 12 & 3 & 28.97 & 2.38 \\
$11 / \mathrm{M}$ & 51 & 40.6 & 20 & 2 & 2 & 25.97 & 2.13 \\
$12 / \mathrm{M}$ & 43 & 31.4 & 25 & 3 & 2 & 28.62 & 1.64 \\
$13 / \mathrm{M}$ & 35 & 40.5 & 10 & 3 & 5 & 27.10 & 2.43 \\
$14 / \mathrm{M}$ & 37 & 28.0 & 10 & 7 & 4 & 27.75 & 2.68 \\
$\mathrm{Mean}$ & 48 & 32.3 & 24 & 6 & 3 & 28.51 & 2.32 \\
$\pm \mathrm{SD}$ & \pm 7 & \pm 4.6 & \pm 11 & \pm 4 & \pm 1 & \pm 1.09 & \pm 0.43
\end{tabular}

$\mathrm{BMI}=$ body mass index, $\mathrm{EDS}=$ excessive daytime sleepiness, $\mathrm{MMSE}=$ mini mental status examination, BMDB: Final result (FR) of Brief Mental Deterioration Battery

$\mathrm{mm} \mathrm{Hg}$ and $71.8 / \mathrm{m}$. All patients presented severe sleep fragmentation with a mean AI of 57 (range 12-81), mean RDI of 58 (range $11-90$ ), mean $\mathrm{SpO}_{2}$ of $86 \pm 4 \%$ (range $77 \pm 7-90 \pm 2$ ), mean nadir $\mathrm{SpO}_{2}$ of $71 \%$ (range 46-88), and oscillations in HR (mean: $64 \pm$ 8 beats/min, range 40-120) and in arterial pressure (mean DBP: $72 \pm 12 \mathrm{~mm} \mathrm{Hg}$, range 46-122; mean SBP: $122 \pm 18.3 \mathrm{mmHg}$, range 77-192). At MSLT all patients had mean sleep latency values $<10$ minutes and sleep onset REM periods (SOREMPs) were seen in 4 patients; one patient had 1 SOREMP, two patients had 2 SOREMPs, and another had 3 SOREMPs (Table 2).

CPAP titration prevented apnoeas, hypopnoeas, and oxyhemoglobin desaturation, and normalized respiratory related arousals and cardiovascular oscillations at a mean nCPAP level of $11 \mathrm{~cm}$ $\mathrm{H}_{2} \mathrm{O}$ (range 8-13). Average sleep latency values at MSLT were higher than baseline, with SOREMP in only one patient.

At CPAP retitration performed after a mean period of 6 months (self-reported estimated use of CPAP $>80 \%$ of total night time), sleep structure and respiratory effort related indices were normal. Mean AI was $5 \pm 3$ (range: 2-9), RDI $1.3 \pm 3$ (range: $0-3$ ) and $\mathrm{SpO}_{2}$ $96 \pm 1$, with fewer cardiovascular oscillations than during diagnostic VPSG (mean HR: $61 \pm 4$ beat/min, range 53-69; mean DBP: $72 \pm 5 \mathrm{mmHg}$, range $62-83$; mean SBP: $121 \pm 7 \mathrm{mmHg}$, range 108136). Mean ESS was $5 \pm 2$ (range: 2-9), mean MSLT was 12 min $8 \mathrm{~s} \pm 4 \mathrm{~min} 40 \mathrm{~s}$ (range: $4 \mathrm{~min} 54 \mathrm{~s}$ to $20 \mathrm{~min}$ ). All parameters reported showed an improvement compared with pre-CPAP values in all cases $(\mathrm{P}<0.001)$.

No patient complained of tiredness or drowsiness, as assessed by means of nonstructured clinical interview, and ESS global score was $\leq 9$, (mean: 5 ; range: $2-9$ ) with a mean BMI of $31 \pm 5$ (not significantly different from pre-CPAP treatment BMI) and mean wake SBP/DBP and HR of 123/81 mm Hg and 69/m.

\section{Neuropsychological Evaluation}

At baseline, all patients had normal general cognitive indexes. When considering the number of pathological neuropsychological tasks (PNT), baseline evaluation detected a small number of PNT $(2.0 \%)$ in 4 patients in tasks exploring immediate verbal and spatial memory and auditory and visual attention tasks; one patient had 3 PNT and one patient 2 PNT. Six months after CPAP, the total number of PNT decreased to $1.1 \%$; patients with PNT were still 4 (two the same as baseline), all with one PNT (almost the same as at baseline) (Table 3). Comparison of the entire OSAS group in the first versus the second evaluations showed significantly improved 15 Rey's words with immediate recall $(\mathrm{P}=0.008)$ and $\mathrm{FR}$ of BMDB $(\mathrm{P}=0.01)$. The same differences were detected when excluding the patients with less severe $(<30)$ RDI.

Table 2-Main baseline and post-CPAP PSG findings from OSAS patients.

\begin{tabular}{|c|c|c|c|c|c|c|c|c|}
\hline \multirow[b]{2}{*}{ Case $n^{\circ}$} & \multicolumn{5}{|c|}{ Baseline } & \multicolumn{3}{|c|}{ Post-CPAP } \\
\hline & RDI & $\begin{array}{l}\text { PSG SpO } \\
\text { mean }(\%)\end{array}$ & $\begin{array}{c}\text { PSG SpO } \\
\text { minimum (\%) }\end{array}$ & $\begin{array}{c}\text { MSLT } \\
\text { (minutes) }\end{array}$ & $\begin{array}{c}\text { ESS } \\
\text { score }\end{array}$ & $\begin{array}{l}\text { CPAP level- } \\
\text { mean } \mathrm{SpO}_{2}\end{array}$ & $\begin{array}{c}\text { MSLT } \\
\text { (minutes) }\end{array}$ & $\begin{array}{c}\text { ESS } \\
\text { Score }\end{array}$ \\
\hline 1 & 62 & $77 \pm 7(56-92)$ & 56 & $3 \min 12 \mathrm{~s}+1 \mathrm{SOREMP}$ & 13 & $8 \mathrm{~cm} \mathrm{H} \mathrm{H}_{2} \mathrm{O}-95 \%$ & $16 \min 30 \mathrm{~s}$ & 4 \\
\hline 2 & 82 & $89 \pm 3(56-94)$ & 56 & 2 min + 2 SOREMPs & 10 & $10 \mathrm{~cm} \mathrm{H}_{2}^{2} \mathrm{O}-96 \%$ & $20 \mathrm{~min}$ & 5 \\
\hline 3 & 43 & $89 \pm 3(79-94)$ & 79 & $9 \min 30 \mathrm{~s}$ & 12 & $8.5 \mathrm{~cm} \mathrm{H}_{2}^{2} \mathrm{O}-95 \%$ & $18 \min 45 \mathrm{~s}$ & 5 \\
\hline 4 & 53 & $87 \pm 4(64-93)$ & 64 & $4 \min 37 s$ & 13 & $8.5 \mathrm{~cm} \mathrm{H}_{2}^{2} \mathrm{O}-96 \%$ & $9 \min$ & 6 \\
\hline 5 & 75 & $83 \pm 5(69-94)$ & 69 & $5 \min 54 \mathrm{sec}+3$ SOREMPs & s 13 & $9 \mathrm{~cm} \mathrm{H}_{2} \mathrm{O}-95 \%$ & $17 \mathrm{~min} 11 \mathrm{~s}$ & 3 \\
\hline 6 & 80 & $80 \pm 7(46-95)$ & 46 & $1 \min 15 \mathrm{~s}$ & 14 & $9 \mathrm{~cm} \mathrm{H}_{2}^{2} \mathrm{O}-95 \%$ & $11 \mathrm{~min}$ & 2 \\
\hline 7 & 90 & $89 \pm 2(80-94)$ & 80 & $4 \min 45 s$ & 12 & $11 \mathrm{~cm} \mathrm{H}_{2}^{2} \mathrm{O}-95 \%$ & $6 \min 37 s$ & 5 \\
\hline 8 & 11 & $90 \pm 1(88-92)$ & 88 & $8 \min 6 s+2$ SOREMPs & 11 & $10 \mathrm{~cm} \mathrm{H}_{2}^{2} \mathrm{O}-96 \%$ & $8 \mathrm{~min}+1$ SOREMP & 5 \\
\hline 9 & 68 & $87 \pm 4(76-94)$ & 76 & $8 \min 30 \mathrm{~s}$ & 13 & $15 \mathrm{~cm} \mathrm{H}_{2}^{2} \mathrm{O}-95 \%$ & $15 \min 30 \mathrm{~s}$ & 9 \\
\hline 10 & 70 & $84 \pm 5(66-94)$ & 66 & $3 \min 52 \mathrm{~s}$ & 13 & $9.5 \mathrm{~cm} \mathrm{H}_{2}^{2} \mathrm{O}-95 \%$ & $11 \mathrm{~min}$ & 4 \\
\hline 11 & 29 & $90 \pm 2(82-94)$ & 82 & $3 \min 42 s$ & 12 & $12 \mathrm{~cm} \mathrm{H}_{2}^{2} \mathrm{O}-96 \%$ & $8 \min 24 \mathrm{~s}$ & 8 \\
\hline 12 & 17 & $88 \pm 3(80-94)$ & 80 & $7 \min 45 s$ & 13 & $10 \mathrm{~cm} \mathrm{H}_{2}^{2} \mathrm{O}-96 \%$ & $10 \mathrm{~min}$ & 4 \\
\hline 13 & 63 & $88 \pm 6(80-94)$ & 80 & $9 \min 30 \mathrm{~s}$ & 18 & $10 \mathrm{~cm} \mathrm{H}_{2}^{2} \mathrm{O}-96 \%$ & $13 \mathrm{~min}$ & 5 \\
\hline 14 & 68 & $84 \pm 5(72-92)$ & 72 & $4 \min 30 s$ & 14 & $9 \mathrm{~cm} \mathrm{H}_{2}^{2} \mathrm{O}-96 \%$ & $4 \min 54 \mathrm{~s}$ & 5 \\
\hline Mean \pm SD & $58 \pm 24$ & $86 \pm 4$ & $71 \pm 12$ & $3 \min 31 \mathrm{~s} \pm 2 \min 44 \mathrm{~s}$ & $13 \pm 2$ & & $12 \min 8 \mathrm{~s} \pm 4 \min 40 \mathrm{~s}$ & $5 \pm 2$ \\
\hline
\end{tabular}




\section{${ }^{1} \mathrm{H}$-MRS Study}

Figure 1B displays representative spectra from an OSAS (case n. 4) and a control subject showing reduced NAA in the patient. Baseline cortical NAA concentration was significantly lower in OSAS patients as a group compared with controls $(\mathrm{P}=0.01)$, whereas no significant difference was found in the $\mathrm{Cr}$ and Cho concentrations (Table 4, Fig. 2). In OSAS patients the cortical [NAA] was positively correlated both with minimum $\mathrm{SpO}_{2}$ during diagnostic VPSG $(\mathrm{r}=0.69 ; \mathrm{P}=0.006)$ and the diagnostic MSLT score $(\mathrm{r}=0.62 ; \mathrm{P}=0.01)$ : patients with lower cortical [NAA] showed lower minimum $\mathrm{SpO}_{2}$ and shorter sleep latency on MSLT (Fig. 3). No correlations were found between any cortical metabolite concentrations and the other clinical and PSG variables (data not shown). After 6 months of CPAP therapy despite the absence of EDS, nocturnal arousals, and $\mathrm{O}_{2}$ desaturations, cortical [NAA], [Cr] and [Cho] were unchanged: cortical [NAA] (post-CPAP) $=11.94 \pm 1.33, \mathrm{P}=0.87 ;[\mathrm{Cr}]($ post-CPAP $)=10.57 \pm 0.94, \mathrm{P}=0.96$; and $[$ Cho $]$ (post-CPAP) $=2.05 \pm 0.15, \mathrm{P}=0.90$.

\section{DISCUSSION}

In our OSAS patients, the baseline PSG demonstrated severe sleep fragmentation and oxygen desaturation, and the MSLT and ESS showed respectively objective and subjective diurnal somnolence. CPAP therapy improved sleep parameters and nearly normalized EDS. One of the limitations of the study was the reliance on purely subjective data when assessing the compliance with CPAP treatment.

At baseline all patients showed normal cognitive general functioning (i.e. normal values of MMSE and FR of BMDB, as shown in Table 1) and only a minority presenting a small number of minor pathological tasks, like working memory (digit span backwards) and some psychomotor tasks (attentional tests) (Table 4). The absence of impairment in general cognitive functioning in our patients may be related to our strict selection criteria for the OSAS sample, in particular the exclusion of patients with cardiovascular, cerebrovascular, or metabolic comorbidities, thus excluding secondary causes of mental deterioration. The neuropsychological tests, especially the Brief Mental Deterioration Battery, employed to evaluate global cognition have shown high specificity and sensitivity in differentiating dementia patients, even those with early onset and short duration of cognitive impairment, from normal controls. ${ }^{24,25}$ There was no suggestion of retest effect on the neuropsychological results after CPAP, and the effect was kept to a minimum by the considerable interval of time elapsing between the 2 evaluations ( 6 months). Ferini-Strambi et $\mathrm{al}^{9}$ failed to find any significant difference between the cognitive test results at intervals of 15 days and 4 months.

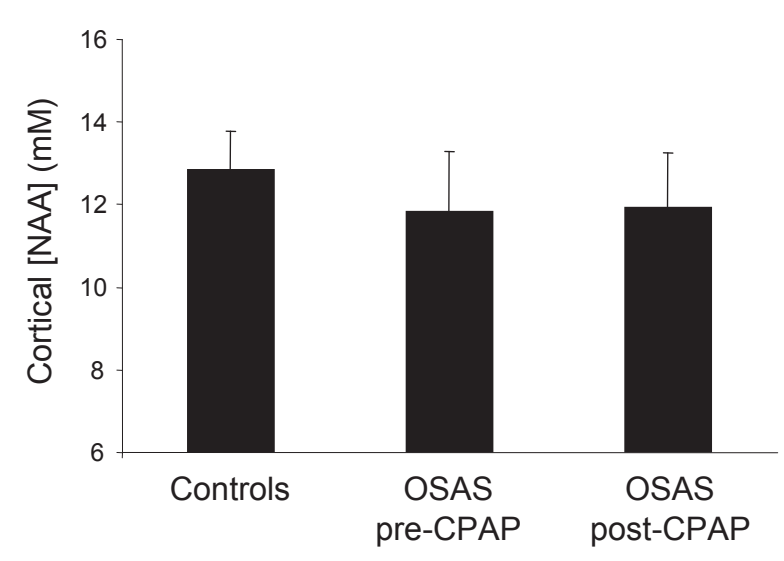

Figure 2-Mean N-acetyl-aspartate (NAA) concentration in the group of OSAS patients pre- and post- CPAP compared to healthy volunteers. Error bars indicate standard deviation.

In contrast with the absence of global cognitive impairment, baseline cortical NAA concentration was lower than in controls and persisted unchanged after therapy despite the improvement of PSG parameters compared to pre-CPAP values in all cases (P $<0.001)$.

In keeping with our findings, MRI and PET/SPECT studies demonstrated brain functional abnormalities in OSAS. Macey et al, using MRI voxel-based morphometry (VBM), found grey matter loss in the frontal, parietal, occipital and temporal cortex, and anterior cingulate gyrus, hippocampus, and cerebellum of 21 OSAS patients. The degree of grey matter loss correlated to disease severity. ${ }^{29}$ Diminished grey matter in the left hippocampus was detected by VBM in a smaller sample of patients. ${ }^{32}$ In contrast, another VBM study failed to detect areas of grey matter volume change in patients with severe OSAS. ${ }^{33}$ A preliminary 18-FDG PET study detected reduced glucose metabolism in the right premotor and anterior cingulate cortical areas,${ }^{34}$ and a preliminary SPECT 99m-TcHMPAO study found reduced perfusion in the temporal-parietal and parietal regions, that improved or normalised after CPAP therapy. ${ }^{35}$

Previous ${ }^{1} \mathrm{H}-\mathrm{MRS}$ studies have thus demonstrated contrasting results, likely related to factors such as different acquisition techniques and localisations prescribed or different patient samples. The ${ }^{1} \mathrm{H}-\mathrm{MRS}$ study of heterogeneous groups of OSAS patients with or without different comorbidities, using the 2-dimensional chemical shift technique, showed a decrease of NAA/Cho, associated with a small reduction in NAA/Cr and increase in Cho/ $\mathrm{Cr}$ in the posterior periventricular white matter. ${ }^{13,36}$ The extent of NAA/Cho reduction was weakly correlated to the apnoea-hypopnoea index. ${ }^{36}$ However, these findings were not confirmed in 2

Table 3 - Pathological Neuropsychological Tasks (PNT) results in OSAS patients before and after CPAP

$\begin{array}{lcccccc} & \begin{array}{c}\text { Total PNT } \\ \mathbf{N}(\%)\end{array} & \begin{array}{c}\text { Case number } \\ \text { with n } \mathbf{1} \mathbf{1} \text { PNT (\%) }\end{array} & \begin{array}{c}\text { Number of pts. } \\ \text { with 3 PNT }\end{array} & \begin{array}{c}\text { Number of pts. } \\ \text { with 2 PNT }\end{array} & \begin{array}{c}\text { Number of pts. } \\ \text { with 1 PNT }\end{array} & \begin{array}{c}\text { Type of task } \\ \text { (number of patients) }\end{array} \\ \text { Baseline } & 7 / 350 & 4,3,9,10 & 1 & 1 & 2 & \text { CRT (2), DSB (1), SS (1), Barr S } \\ \text { After CPAP } & (2.0 \%) & (28.5 \%) & & & 4 & \text { (1), CVART (1), CVARTe (1) } \\ & 4 / 350 & 4,3,11,12 & 0 & 0 & \text { CRT (1), SRTe (1), DSB (1), } \\ & (1.1 \%) & (28.5 \%) & & & \text { Barr S (1), SVARTe (1) }\end{array}$

CRT: complex reaction times; DSB: digit span backwards; SS: spatial span; Barr S: barrage score; CVART: complex visual reaction times; CVARTe: complex visual reaction times errors; SRTe: simple reaction time errors; SVARTe: simple visual attention reaction times errors. 

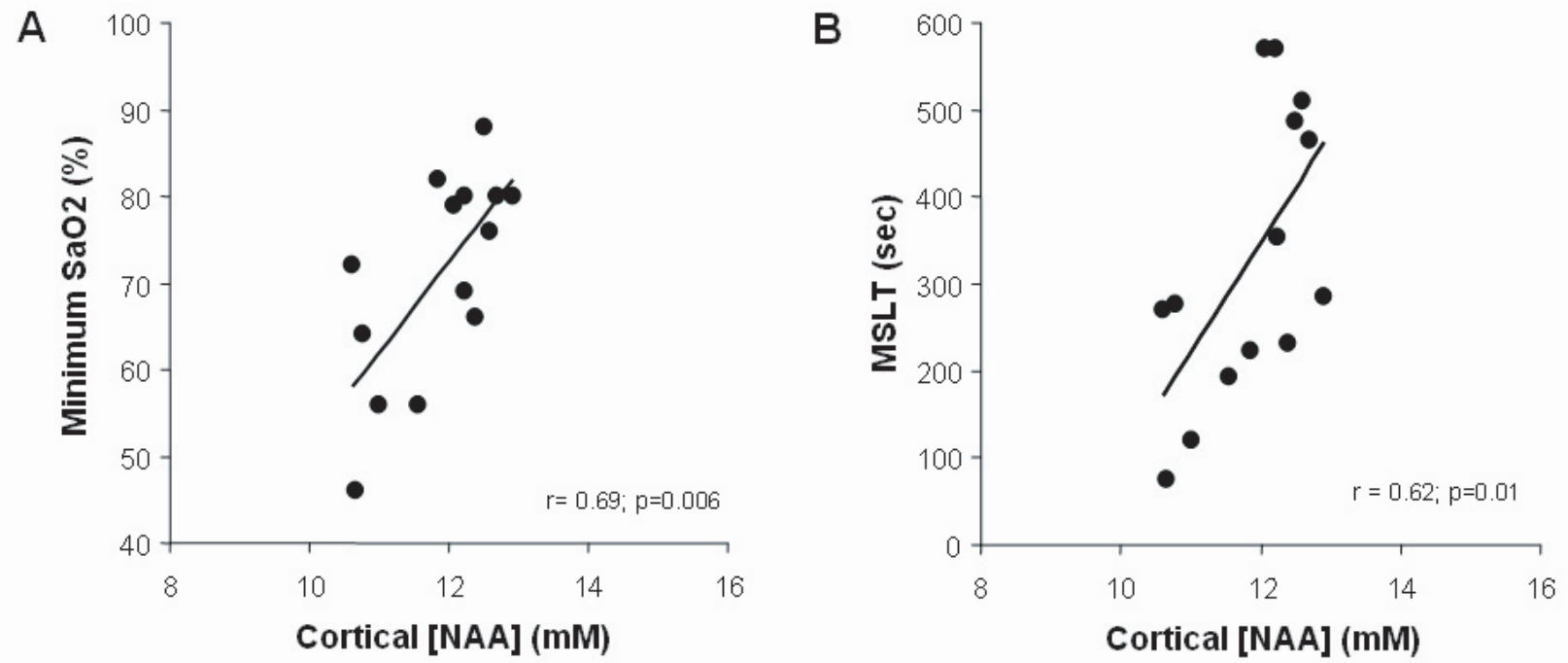

Figure 3 - Correlation between cortical N-acetyl-aspartate (NAA) concentration and minimum $\mathrm{SpO}_{2}$ during nocturnal sleep (A) and MSLT score (B) in OSAS patients.

later ${ }^{1} \mathrm{H}$-MRS single-voxel studies of OSAS patients that pointed either to a reduction in NAA and Cho (frontal white matter) ${ }^{14}$ or in Cr content (left hippocampus). ${ }^{15}$ Most ${ }^{1} \mathrm{H}$-MRS studies undertook no neuropsychological assessment and post-therapy re-examination to verify the potential reversibility of biochemical changes.

Our ${ }^{1} \mathrm{H}$-MRS results indicate that in OSAS subjects the concentration of cortical NAA may be reduced in the absence of structural lesions, as assessed by means of brain MRI obtained before and after 6 months of CPAP treatment in each patient, and before the development of major cognitive impairment. The persistence of low cortical NAA concentration after therapy indicates a neuronal loss rather than a neuronal dysfunction, most likely related to the repeated episodes of hypoxia. Interestingly, high brain lactate has been detected using ${ }^{1} \mathrm{H}-\mathrm{MRS}$ in some OSAS patients studied during sleep. ${ }^{37}$

The role of hypoxia in determining cortical damage is support-

Table 4-Cortical metabolite concentrations ( $\mathrm{mmol} / \mathrm{Kg}$ wet weight) in pre-CPAP OSAS patients and in healthy controls.

\begin{tabular}{|c|c|c|c|}
\hline $\operatorname{Case} n^{\circ}$ & [NAA] & {$[\mathbf{C r}]$} & [Cho] \\
\hline 1 & 11.55 & 11.37 & 2.10 \\
\hline 2 & 11.01 & 11.09 & 1.94 \\
\hline 3 & 12.07 & 10.65 & 1.98 \\
\hline 4 & 10.78 & 10.39 & 2.01 \\
\hline 5 & 12.23 & 11.69 & 2.19 \\
\hline 6 & 10.67 & 9.02 & 1.84 \\
\hline 7 & 12.92 & 12.76 & 2.02 \\
\hline 8 & 12.51 & 10.55 & 2.15 \\
\hline 9 & 12.59 & 9.37 & 1.99 \\
\hline 10 & 12.38 & 10.99 & 2.07 \\
\hline 11 & 11.85 & 10.56 & 2.18 \\
\hline 12 & 12.70 & 10.63 & 2.33 \\
\hline 13 & 12.23 & 10.64 & 1.74 \\
\hline 14 & 10.61 & 7.97 & 2.08 \\
\hline Mean \pm SD & $11.86 \pm 0.80$ & $10.55 \pm 1.17$ & $2.04 \pm 0.15$ \\
\hline Controls (n. $=10)$ & $12.85 \pm 0.93$ & $10.58 \pm 0.77$ & $2.13 \pm 0.17$ \\
\hline$P$ values & 0.01 & NS & NS \\
\hline
\end{tabular}

ed by a number of experimental models. ${ }^{38,39}$ Exposure to intermittent hypoxia, like that occurring in OSAS, led to neurobehavioral impairment in the absence of significant sleep disruption..$^{38}$ Intermittent hypoxia in developing rat brain is associated with cognitive deficits like impaired spatial learning and hyperactivity ${ }^{39}$ similar to the clinical findings observed in some pediatric OSAS patients. ${ }^{40}$ The neurobehavioral deficits of adult rats are correlated to degenerative changes in the cortex and hippocampus. ${ }^{38}$

Our study showed that the extent of cortical neuronal damage in OSAS patients was consistently related to the minimum $\mathrm{SpO}_{2}$ occurring during nocturnal sleep. The absence of any other significant correlations between cortical NAA reduction and other clinical and VPSG variables further supports the role of intermittent hypoxia, rather than arousals and sleep fragmentation, on the metabolic cerebral changes observed in our patients.

In conclusion, the cortical NAA reduction found in our sample of OSAS patients without cardiovascular disease supports the central pathogenetic role of cerebral hypoxia in obstructive apnoea. This biochemical alteration, unchanged after effective CPAP therapy, suggests irreversible cerebral damage in OSAS patients. To elucidate the natural history of brain involvement in the sleep apnoea syndrome, longitudinal ${ }^{~} \mathrm{H}-\mathrm{MRS}$ studies of patients from the early stages of the disease will help to define the window of reversibility of metabolic changes in the brain.

\section{ACKNOWLEDGEMENTS}

This work received grants MIUR from COFIN 2000.

\section{REFERENCES}

1. Young T, Palta M, Dempsey J, et al. The occurrence of sleep disordered breathing among middle aged adults. N Engl J Med 1993;328:1230-5.

2. Ancoli-Israel S, Klauber MR, Stepnowsky C, Estline E, Chinn A, Fell R. Sleep-disordered breathing in African-American elderly. Am J Respir Crit Care Med 1995;152:946-9.

3. American Academy of Sleep Medicine. Obstructive sleep apnea syndromes. In: The International classification of sleep disorders, $2^{\text {nd }}$ ed.: Diagnostic and coding manual. Westchester, IL: American Academy of Sleep Medicine, 2005:51-62. 
4. Lavie L. Sleep-disordered breathing and cerebrovascular disease: a mechanistic approach. Neurol Clin 2005;23:1059-75.

5. Aloia MS, Arnedt JT, Davis JD, Riggs RL, Byrd D. Neuropsychological sequelae of obstructive sleep apnea-hypopnea syndrome: a critical review. Int Neuropsychol Soc 2004;10:772-85.

6. Beebe DW, Groesz L, Wells C, Nichols A, McGee K. The neuropsychological effects of obstructive sleep apnea: a meta-analysis of norm-referenced and case-controlled data. Sleep 2003;26:298-307.

7. Mansfield DR, Gollogly NC, Kaye DM, Richardson M, Bergin P, Naughton MT. Controlled trial of continuous positive airway pressure in obstructive sleep apnea and heart failure. Am J Respir Crit Care Med 2004;169:361-6.

8. McMahon JP, Foresman BH, Chisholm RC. The influence of CPAP on the neurobehavioral performance of patients with obstructive sleep apnea hypopnea syndrome: a systematic review. WMJ 2003;102:36-43.

9. Ferini-Strambi L, Baietto C, Di Gioia MR, et al. Cognitive dysfunction in patients with obstructive sleep apnea (OSA): partial reversibility after continuous positive airway pressure (CPAP). Brain Res Bull 2003;61:87-92.

10. Rudkin TM, Arnold DL. Proton magnetic resonance spectroscopy for the diagnosis and management of cerebral disorders. Arch Neurol 1999;56:919-26.

11. Narayanan S, De Stefano N, Francis GS, et al. Axonal metabolic recovery in multiple sclerosis patients treated with interferon beta- $1 \mathrm{~b}$. J Neurol 2001;248:979-86.

12. De Stefano N, Matthews PM, Arnold DL. Reversible decreases in N-acetylaspartate after acute brain injury. Magn Reson Med 1995;34:721-7.

13. Kamba M, Suto Y, Ohta Y, Inoue Y, Matsuda E. Cerebral metabolism in sleep apnea. Evaluation by magnetic resonance spectroscopy. Am J Respir Crit Care Med 1997;156:296-8.

14. Alchanatis M, Deligiorgis N, Zias N, et al. Frontal brain lobe impairment in obstructive sleep apnoea: a proton MR spectroscopy study. Eur Respir J 2004;24:980-6.

15. Bartlett DJ, Rae C, Thompson CH, et al. Hippocampal area metabolites relate to severity and cognitive function in obstructive sleep apnea. Sleep Med 2004;5:593-6.

16. Keevil SF, Barbiroli B, Brooks JC, et al. Absolute metabolite quantification by in vivo NMR spectroscopy: II. A multicentre trial of protocols for in vivo localised proton studies of human brain. Magn Reson Imaging 1998;16:1093-106.

17. Rechtschaffen A, Kales A. A manual of standardized terminology, techniques and scoring system for sleep stages of human subjects. Los Angeles: Brain Information Service, Brain Research Institute, 1968.

18. Guilleminault C, van den Hoed J, Mitler MM. Clinical overview of the sleep apnea syndromes. In: Guilleminault C, Dement WC, eds. Sleep apnea syndromes. New York: Alan R. Liss, 1978:1-12.

19. Block AJ, Boysen PG, Wynne JM, Hunt LA. Sleep apnea, hypopnea and oxygen desaturation in normal subjects. A strong male predominance. N Engl J Med 1979;300:513-17.

20. Kryger MH. Monitoring respiratory and cardiac function. In: Kryger M, Roth T, Dement W, eds. Principles and practice of sleep medicine. 3rd ed. Philadelphia: W.B. Saunders Company, 2000:121730 .

21. Carskadon MA, Rechthschaffen A. Monitoring and staging human sleep. In: Kryger M, Roth T, Dement W, eds. Principles and practice of sleep medicine. 3rd ed. Philadelphia: W.B. Saunders Company, 2000:1197-215.

22. Carskadon MA, Dement WC, Mitler MM, Roth T, Westbrook PR, Keenan S. Guidelines for the multiple sleep latency test (MSLT): a standard measure of sleepiness. Sleep 1986;9: 519-24.

23. Johns MW. A new method for measuring daytime sleepiness: the Epworth sleepiness scale. Sleep 1991;14:540-5.

24. Folstein MF, Folstein SE, McHugh PR. "Mini-mental state". A practical method for grading the cognitive state of patients for the clini- cian. J Psychiatr Res 1975;12:189-98

25. Measso G, Cavarzeran F, Zappala G et al. The Mini-Mental State Examination: normative study of an Italian random sample. Dev Neuropsychol 1993;9:77-85.

26. Gallassi R, Lenzi P, Stracciari A, et al. Neuropsychological assessment of mental deterioration: purpose of a brief battery and a probabilistic definition of "normality" and "non-normality". Acta Psychiatr Scand 1986;74:62-7.

27. Gallassi R, Morreale A, Di Sarro R, Lorusso S. Value of clinical data and neuropsychological measures in probable Alzheimer's disease. Arch Gerontol Geriatr 2002;34:123-34.

28. Gallassi R, Morreale A, Montagna P et al. Fatal familial insomnia: behavioral and cognitive features. Neurology 1996;46:935-9.

29. Macey PM, Henderson LA, Macey KE, et al. Brain morphology associated with obstructive sleep apnea. Am J Respir Crit Care Med 2002;166:1382-7.

30. De Beer R, Barbiroli B, Gobbi G et al. Absolute metabolite quantification by in vivo NMR spectroscopy: III. Multicentre ${ }^{1} \mathrm{H}$ MRS of the human brain addressed by one and the same data-analysis protocol. Magn Reson Imaging 1998;16:1107-11.

31. Lodi R, Tonon C, Vignatelli L, et al. In vivo evidence of neuronal loss in the hypothalamus of narcoleptic patients. Neurology 2004;63:1513-5.

32. Morrell MJ, McRobbie DW, Quest RA, Cummin AR, Ghiassi R, Corfield DR. Changes in brain morphology associated with obstructive sleep apnea. Sleep Med 2003;4:451-4.

33. O'Donoghue FJ, Briellmann RS, Rochford PD, et al. Cerebral structural changes in severe obstructive sleep apnea. Am J Respir Crit Care Med 2005;171:1185-90.

34. Pietrini P, Dani A, Raphaelson M, et al. Cerebral glucose metabolic and neuropsychological dysfunction in patients with untreated sleep apnoea syndrome (SAS). Sleep 1998;21:82.

35. Feistel H, Merkl M, Siegfried W, et al. Brain perfusion during sleep apnea. A study with TC-99M-HMPAO in sleep laboratory. Eur J Nucl Med 1994;21:770.

36. Kamba M, Inoue Y, Higami S, Suto Y, Ogawa T, Chen W. Cerebral metabolic impairment in patients with obstructive sleep apnoea: an independent association of obstructive sleep apnoea with white matter change. J Neurol Neurosurg Psychiatry 2001;71:334-9.

37. Kamba M, Inoue Y, Higami S, Suto Y. Age-related changes in cerebral lactate metabolism in sleep-disordered breathing. Neurobiol Aging 2003;24:753-60.

38. Gozal D, Daniel JM, Dohanich GP. Behavioral and anatomical correlates of chronic episodic hypoxia during sleep in the rat. J Neurosci 2001;21:2442-50.

39. Row BW, Kheirandish L, Neville JJ, Gozal D. Impaired spatial learning and hyperactivity in developing rats exposed to intermittent hypoxia. Pediatr Res 2002;52:449-53.

40. Gozal D, Pope DW Jr. Snoring during early childhood and academic performance at ages thirteen to fourteen years. Pediatrics 2001;107:1394-9. 